\title{
PONENCIA
}

\section{Un acercamiento al diálogo entre la educación técnica agropecuaria e industrial y el sector productivo}

\author{
Alcira Sosa1', Sara Raquel López¹, María Victoria Zavala1, Hugo \\ Speratti ${ }^{1}$, Graciela Molinas ${ }^{1}$ \\ Asesoría: Claudio Suasnabar, Rudy Elías
}

\begin{abstract}
Ante este escenario, nos permitimos asumir como objeto central del trabajo el acercar el avance de la investigación en curso sobre el diálogo entre la Educación Técnica Agropecuaria e Industrial y el Sector Productivo,con la prudencia y el respeto que nos inspira todo el esfuerzo que dichos sectores imprimen en el trabajo por lograr los objetivos propuestos, situación apreciada en las visitas en las que nos permitieron aplicar los instrumentos de recogida de datos, como entrevistas individuales y grupales.

Iniciaremos exponiendo los aspectos relacionados a la educación, tanto en lo declarativo en los documentos oficiales del Sistema Educativo, en el Nivel de la Educación Media, en Bachilleratos Técnicos Agropecuarios e Industrial de la Educación Media, posteriormente se presentará los aspectos relacionados al sector productivo y los testimonios representantes de empresas entrevistados para concluir con las reflexiones que generan los primeros hallazgos de la investigación.

Entre las variadas maneras de concebir la educación y a fin de sustentar en ella las ideas expuestas en este trabajo, se ha seleccionado las siguientes:
\end{abstract}

DEWEY (1859-1952)²: La educación es la suma total de procesos por medio de los cuales una comunidad o un grupo social pequeño o grande transmite su capacidad adquirida y sus propósitos con el fin de asegurar la continuidad de su propia existencia y desarrollo.

1. Universidad Iberoamericana, Paraguay.

Correspondencia: Graciela Molinas. E-mail: gracielamspy@gmail.com

Presentado en el I Congreso Paraguayo de Ciencias Sociales. "Las ciencias sociales ante los retos de la justicia social", 11 al 17 de julio de 2017. Asunción, Paraguay.

Este trabajo se enmarca en las reflexiones preliminares surgidas en el proceso de desarrollo de la investigación denominada "Formación para el trabajo, en busca de la pertinencia de los Bachilleratos Técnicos Agropecuarios e Industrial de la Educación Media", que cuenta con el apoyo del Consejo Nacional de Ciencia y Tecnología, CONACYT; se pretende describir la relación entre las competencias laborales demandadas por el sector productivo del país y la formación en Bachilleratos Técnicos Agropecuarios e Industrial de la Educación Media.

2 John Dewey: filósofo, pedagogo y psicólogo estadounidense. Nacido en Burlington, Vermont, 20 de octubre de 1859-Nueva York, 1 de junio de 1952. Asimismo, durante la primera mitad del siglo XX fue la figura más representativa de la pedagogía progresista en Estados Unidos.

Recibido: 25/06/2017. Aceptado: 17/07/2017.

DOI: 10.26885/rcei.6.1.78 


\section{Educación técnica agropecuaria e industrial y el sector productivo. Sosa et al.}

DURKHEIM (1858-1917)3: La educación tiene por misión desarrollar en el educando los estados físicos intelectuales y mentales que exigen de él la sociedad política y el medio social al que está destinado.

HUBERT (1885-1954)4: "La educación es el conjunto de las acciones y de las influencias ejercidas voluntariamente por un ser humano sobre otro; en principio, por un adulto sobre un joven, y orientados hacia un objetivo que consiste en la formación juvenil de disposiciones de toda índole correspondiente a los fines para los que está deudo, tría vez ${ }^{5}$ que llegue a su madurez.

En las concepciones de educación expuestas, se puede identificar la idea de una acción sistemática, procesual y provocada por el hombre ${ }^{6}$ con el objetivo de incidir en la formación de las diferentes generaciones, a través de la transmisión de conocimientos sistematizados que tenderían a incidir de manera individual y colectiva en las personas; tanto para el desarrollo de la sociedad como para el logro de madurez de los individuos. Además, se puede inferir que el surgimiento de los sistemas educativos, en los estados nación probablemente se hayan desarrollado en respuesta a estas ideas de educación.

Teniendo como referencia, el Sistema Educativo Paraguayo, en el marco de los objetivos de la educación paraguaya podemos identificar, entre varios otros objetivos, uno de ellos que hace referencia específica a la intencionalidad de la formación técnica en el trayecto académico en los bachilleratos del nivel de la educación media, tanto para la educación técnica como la científica: "dar formación técnica a los educandos en respuesta a las necesidades de trabajo y a las cambiantes circunstancias de la región y del mundo" (MEC, 1993), este anhelo definido en el Diseño Curricular Nacional, induce a la idea de que la educación ofrecida en las instituciones de educación media, distribuidas por todo el país, tendrían que dar respuestas a dichas necesidades y a la vez dinamizarse a partir de los cambios emergentes en la sociedad.

La educación, tal como se la concibe como uno de los principios fundamentales para el desarrollo de un país, ya que una persona adecuadamente formada según la propuesta definida en el Diseño Curricular Nacional, sería una persona que tendría las capacidades, habilidades y actitudes para contribuir al progreso del país, en el lugar donde se desempeñe.

Cuando nos remitimos a la educación técnica, estrechamente vinculado al sector productivo, es más que determinante que la formación debería responder a las necesidades específicas del mercado laboral, las cuales cambian con el tiempo y a su formación general para un desempeño asertivo en los ámbitos de actuación. Por ello, se hace oportuno lo expuesto por Filmus (2001), al decir que

3 Émile Durkheim. Sociólogo, pedagogo y antropólogo francés, uno de los pioneros en el desarrollo de la moderna sociología.

4 Hubert, René. Filósofo y pedagogo francés, fue profesor en la universidad de Estrasburgo.

5 Según la Real Académica Española, diccionario usual: es, escoger, separar, entresacar.

6 En su concepción más genérica de concebirla. 


\section{Rev. cient. estud. investing. 6(1), 78-87; setiembre 2017}

La forma de articulación de la educación con este mercado, en particular de la escuela media, se centraría en la necesidad de capacitar a sus alumnos para el desempeño de los modernos puestos de trabajo. Desde estas posiciones, la principal responsabilidad de la falta de articulación estaría radicada en el sistema educativo, que atrasa respecto de las nuevas demandas de formación que el empleo requiere en la actualidad. ( p. 149)

La evolución tecnológica, la producción en general, ha posibilitado elevar los niveles de competitividad de las empresas, las condiciones de vida y de trabajo de la población, requiriendo mejorar permanentemente la calidad y pertinencia de la educación y la formación de los recursos humanos. Con el proceso de Reforma Educativa en la educación media, en el año 2002 se realizan ajustes a los diseños curriculares que no modifican substancialmente la oferta del nivel (MEC, 2011) y el bachillerato técnico queda distribuidos en sector industrial, sector agropecuario, sector servicios.

La falta de atención a las funciones educativas, económicas y sociales de la educación media deja de lado su propia identidad y carente de estrategias pertinentes para enfrentar los desafíos que el contexto social y productivo presenta a los jóvenes. Cabe resaltar, lo planteado por María Eugenia Gallart al señalar que la educación y el trabajo son dos racionalidades distintas "sin embargo, la escuela debe estar en condiciones de desarrollar en los y las estudiantes capacidades básicas -valores, conocimientos y habilidades- que permitan adaptarse y hacer un aprendizaje de los cambios que ocurren" (María Eugenia Gallart, comunicación personal, 2003).

El Plan Nacional de Mejoramiento de la Educación Técnica y Profesional en el Paraguay 2011-2013, realizó un estudio que permite conocer relación entre oferta y demanda de la Formación Técnica Profesional, según departamentos, en la cual se propone abordar, entre otros, uno de los nudos críticos relacionados con la insuficiente pertinencia de la formación para responder a requerimientos del sector productivo, en cuyo ámbito cae esta investigación. Cabe resaltar que la implementación del abordaje de la educación para el trabajo en la educación media implica el análisis crítico de la situación y características de este nivel educativo (Messina, Pieck \& Castañeda, 2008) para develar las carencias y debilidades de la política pública al respecto.

El estudio de seguimiento a egresados y egresadas de la educación media técnica, bachillerato técnico en contabilidad, bachillerato técnico agropecuario, bachillerato técnico en lectricidad (MEC, 2015), da cuenta en sus conclusiones, que si bien no son concluyentes permite acercar unas líneas de reflexión en torno a la oferta educativa de los bachilleratos técnicos y la demanda laboral existente, entre otros aspectos como las siguientes:

La compleja relación escuela - trabajo se encuentra atravesada por niveles de tensión en el cual el modelo productivo actual con sus consecuentes repercusiones no acompañan los procesos de formación de la educación secundaria y viceversa. 


\section{Educación técnica agropecuaria e industrial y el sector productivo. Sosa et al.}

Es necesaria dejar instalada la reflexión en torno a la relación entre oferta de los bachilleratos técnicos (en términos de inversión y expansión) y la demanda actual y potencial teniendo como base en primer lugar los planes de desarrollo nacional y departamental. ()

Las ideas expuestas del estudio mencionado se pudieron constatar, en los primeros hallazgos obtenidos, en contacto con representantes del sector productivo, se ha podido percibir dicha tensión, como en el siguiente testimonio:

la empresa se hace cargo de la formación de sus recursos humanos, porque sería algo poco probable que cuente con egresados de la Educación Técnica. Tuvimos uno en una ocasión, lo que pudimos ver es que tenía los conocimientos básicos para entender lo que se esperaba de él y resultó más fácil la capacitación; con los otros que son la mayoría, hay que empezar de cero. (Entrevista a empresario de tecnología de la comunicación)

En relación a la manera de concebir la formación de profesionales es menester incorporar la perspectiva del curriculum que pasa por una revisión de concepciones como el planteado por Stenhouse (1987) que considera que es un medio con el cual se hace públicamente disponible la experiencia consistente en intentar poner en práctica una propuesta educativa. Con una posición más social, Jurjo Torres ( 2002) lo describe como un proyecto educativo que se planifica y desarrolla a partir de una selección de la cultura y de las experiencias en las que se desea que participen las nuevas generaciones con el fin de socializarlas y capacitarlas para ser ciudadanos y ciudadanas solidarias, responsables y democráticas. Lo común de las concepciones presentadas remiten a la idea de selección de saberes considerados válidos para el aprendizaje que orientan las acciones pedagógicas en los centros educativos, dichos saberes se explicitan en "instrumentos oficiales que se consideren legítimos y principales a través del cual (o de los cuales), en cada país o región, el Estado ordena las prácticas de enseñanza y determina cual es el saber oficializado" (Gvirtz \& Palamidessi, 2004, p. 75).

Así como hemos evolucionado de "la formación de la mano de obra calificada" (que reducía la capacidad humana en el ámbito laboral, a una actividad productiva mecánica, casi incuestionable) al reconocimiento del "capital humano", es importante reconocer la influencia de estas definiciones del ámbito de la economía como variables que han incidido en la construcción social del valor del conocimiento que las personas acumulamos, más allá de otras determinantes de tipo social que durante años han limitado el derecho a la educación a condicionantes de segregación según las clases sociales, enajenando incluso las oportunidades de una formación continua y postergando las posibilidades de las personas de mejorar su calidad de vida.

Probablemente, en la relación sociedad-educación-economía, en las últimas décadas se han reconfigurado las representaciones en un diálogo más horizontal que nos permite conversar en perspectivas que van más allá de una 


\section{Rev. cient. estud. investing. 6(1), 78-87; setiembre 2017}

dependencia unilateral, donde por ejemplo ya no se concibe a la educación como subsumida a la economía y la entendemos como un derecho humano fundamental, con sentido en sí misma y como oportunidad de desarrollo integral de las personas y por ende, de la sociedad en su conjunto.

Sin dudas el desarrollo económico, posibilita el desarrollo social, cuando la distribución de los bienes y servicios, garantiza la mejora de las condiciones de vida de las personas, y ello supone en el análisis del estudio referido identificar cómo se articulan el ámbito educativo y el ámbito laboral. Pensar en la categoría de articulación, supone salirse del discurso de la dependencia de uno en relación al otro, sobre todo cuando ciertas tendencias consideran que "uno forma para el otro", y que todo el sistema educativo debería pensarse en base a las exigencias del sistema productivo. De alguna manera, también otras tendencias han demostrado que la formación del capital humano, ya no es una cuestión predecible y posible de determinar para el largo plazo (por la celeridad de los cambios de la sociedad del conocimiento, los avances tecnológicos, las dinámicas sociales, entre otros), por lo cual en ambos ámbitos-educativo y productivo- se reconoce la necesidad de una educación/formación a lo largo de la vida.

Esta educación a lo largo de la vida, que sin dudas trae aparejado varios desafíos, permite definir una nueva concepción de la formación en ambos ámbitos, tanto en términos de abordajes curriculares, estructuras de reconocimiento de saberes y sus sistemas de promoción, organización institucional e incluso aspectos de infraestructura e innovación, entre otros. Este nuevo enfoque redefine las cuestiones de la formación general y la formación específica, tanto en el espacio de la institución educativa como en el laboral, conjugando además aspectos culturales, científicos y tecnológicos con mayor o menor profundización.

Asumiendo la definición de pertinencia como adecuación, conveniencia, indagamos en las condiciones que en el periodo de estudio definen la oferta de los bachilleratos técnicos, de los sectores industrial y agropecuario, en relación con el desarrollo productivo/laboral en la perspectiva de identificar los aspectos de adecuación conveniente que garanticen a los educandos del nivel medio mejores oportunidades de formación.

El estudio indaga algunas características de los procesos de formación en el nivel medio en instituciones del sector oficial y privado subvencionado, con ofertas de bachillerato técnico agropecuario y bachillerato técnico industrial.

La cuestión central de la investigación se ancla en la relación ofertademanda considerando las similitudes y diferencias entre las competencias desarrolladas en las instituciones educativas y las requeridas en el sector productivo. También se consideró importante identificar el conocimiento y motivación del estudiante en relación a la especialidad elegida, dado que no todas las instituciones cuentan con servicio de orientación vocacional que asesore a los estudiantes en la elección de una carrera técnica.

En relación a los docentes se recoge información sobre el perfil en 


\section{Educación técnica agropecuaria e industrial y el sector productivo. Sosa et al.}

términos de su formación inicial y de las variaciones de dicho perfil considerando las especialidades que enseña. Y esto constituye una de las debilidades iniciales de la política pública en el ámbito de la formación docente, dado que no existen instituciones especializadas para la formación orientada al sector técnico industrial y agropecuario. Por tanto, ejercen la docencia mayoritariamente, especialistas de las áreas con título de grado, pero sin habilitación pedagógica para el ejercicio de la docencia, o en su defecto técnicos de nivel terciario, también sin habilitación pedagógica. El sistema contempla cursos denominados de habilitación pedagógica, que posibilitan la formación didáctico pedagógica y el acceso a la carrera docente mediante concursos públicos de oposición. En términos de percepción se indaga sobre los niveles de satisfacción de los mismos en el ejercicio de la docencia y las expectativas que los estudiantes tienen al respecto de sus docentes.

En cuanto a la percepción sobre la oferta formativa, que tienen los directores, docentes y estudiantes, se organiza en los siguientes ámbitos y objetivos:

\section{Tabla 1}

\section{Percepción de la oferta educativa}

\begin{tabular}{ll}
\hline ÁMBITOS & OBJETIVOS \\
\hline Curriculum & $\begin{array}{l}\text { Recoger la opinión sobre la organización curricular del } \\
\text { bachillerato Recoger la opinión sobre las expectativas } \\
\text { del estudiante sobre la especialidad elegida. }\end{array}$ \\
\hline Metodología & $\begin{array}{l}\text { Identificar las técnicas didácticas utilizadas en la } \\
\text { institución. } \\
\text { Establecer grado de coincidencias/diferencias entre los } \\
\text { grupos entrevistados en relación a la técnicas didácticas } \\
\text { utilizadas. }\end{array}$ \\
\hline Rvaluación & $\begin{array}{l}\text { Recoger percepción/opinión sobre el sistema de } \\
\text { evaluación vigente. } \\
\text { Conocer los niveles de logro educativo de los estudiantes. } \\
\text { Identificar mecanismos de acompañamiento. } \\
\text { institucional a los procesos evaluativos. }\end{array}$ \\
\hline Infraestructura & $\begin{array}{l}\text { Identificar si las condiciones de infraestructura. } \\
\text { responden a las necesidades de formación. }\end{array}$ \\
\hline
\end{tabular}

Nota. Elaboración propia a partir del instrumento para entrevistas a actores educativos.

En el análisis de los ámbitos, se identifica que, en el marco de las reformas curriculares, con más o menos aciertos, se han delineado ajustes en el enfoque curricular, la metodología y el sistema de evaluación, que en términos de diseño y propuesta probablemente recogen algunas tendencias de la región, pero no se ha previsto en términos de política pública, una intervención 


\section{Rev. cient. estud. investing. 6(1), 78-87; setiembre 2017}

gradual e integral que garantice las condiciones para su implementación. Es decir, no existe una oferta de formación de docentes como ya mencionamos y la infraestructura de las instituciones educativas ha permanecido inmutable al paso del tiempo, sin adecuaciones físicas ni tecnológicas que permitan el acceso a talleres y laboratorios propios de cada especialidad, por mencionar algunos aspectos.

Una cuestión no menor, es la no existencia de línea presupuestaria que contemple los recursos para la inversión en infraestructura de colegios técnicos, por lo cual resulta muy poco pertinente habilitar el funcionamiento de instituciones educativas sin las mínimas condiciones requeridas, dejando librados a su suerte a los estudiantes que acuden a instituciones donde la formación técnica se agota en una formación teórica. Como ejemplo se menciona lo que se pudo comprobar en una institución del Este del país, en la que los estudiantes del bachillerato Técnico Agropecuario, solo contaban con un espacio de $12 \mathrm{~m}$ por $30 \mathrm{~m}$, para realizar cultivos que se limitaban a una huerta, ubicada a dos cuadras del colegio, en una zona en la que se visualiza la prevalencia de la agricultura masificada. Y esto es una cuestión que debiera tomarse con las debidas precauciones porque incide en la precarización de la oferta educativa y de los logros de aprendizaje. En el comparativo de la percepción de logros académicos, aunque resulte obvio comentarlo, las diferencias están marcadas por la condición de la infraestructura y por el perfil de los docentes en un alto porcentaje, como aspectos de mayor incidencia.

Cabe resaltar que en las visitas realizadas en los colegios que ofrecen estos bachilleratos, se pudo constatar que en algunas instituciones se generan acciones de autogestión, como el caso de una institución del Sur del país, que manifestaban las estrategias que realizaban para disponer de máquinas que requieren para la enseñanza especializada, en el bachillerato técnico en automotriz:

nosotros lo que hacemos es contactar con la comisaría para solicitar que nos concedan, los motores automáticos de los autos chocados que no son retirados del lugar y hasta ahora ellos colaboran con nosotros, mediante eso podemos enseñar las características de estos motores porque lo que tenemos en el taller son solo los mecánicos. (Entrevista grupal a docentes)

Desde la experiencia del sector productivo, se recogen percepciones variadas determinadas tanto por los niveles de especialización de cada ramo, como por las referencias construidas sobre diferencias en el perfil de los egresados en relación a las instituciones en que se formaron.

Una cuestión interesante pasa por identificar, en términos de tipología, ciertas características que definen los procesos de incorporación de los egresados de nivel medio en empresas de pequeño y mediano tamaño, considerando que no siempre éstas disponen de estrategias de captación de personal, salvo por la vacancia o por la necesidad de la expansión (a veces concentrada en ciertas temporadas). Y en esto también sin dudas incide la 


\section{Educación técnica agropecuaria e industrial y el sector productivo. Sosa et al.}

percepción que tiene el sector productivo sobre las competencias de los egresados del nivel medio, y en algunos casos particularmente según la proveniencia de una u otra institución educativa.

Otro aspecto del diálogo pasa por el tema de pasantías, que no siempre tiene un andamiaje entre los sectores educativo y productivo, en la generalidad de lo recabado se reduce al cumplimiento de unas horas y el reporte de unas actividades básicas que no conllevan un valor agregado a la formación de los futuros técnicos. Explorar con mayor prospectiva este tema, puede generar un escenario de oportunidades para ambos sectores, considerando que en relación al desarrollo de competencias y la incorporación de tecnología, el sector productivo tiene mejores condiciones y que pensado coordinadamente este espacio puede representar una experiencia enriquecedora para ambos.

Una estrategia que puede acercar las oportunidades del sector productivo a las condiciones de funcionamiento de las instituciones educativas se observa tímidamente en las iniciativas de Responsabilidad Social, aún muy incipiente en el sector, con contadas menciones en las entrevistas.

No se dispone aún de información sistematizada sobre las competencias generales ni sobre las competencias específicas que se estiman necesarias en el sector productivo, que posibiliten un análisis en relación a las competencias definidas en lo lineamientos curriculares del nivel.

\section{RefERencias}

Filmus, D. (2001). La Educación Media frente al mercado de trabajo. Cada vez más necesaria, cada vez más insuficiente trabajo (p. 149-222). En C. Braslavsky (Ed.). La educación secundaria ¿cambio o inmutabilidad. Análisis y debates de procesos europeos y latinoamericanos. Buenos Aires: UNESCO. Santillana.

Gvirtz, S. \& Palamidessi, M. (2004). El ABC de la tarea docente: currículum y enseñanza. Buenos Aires: Aique.

Torres, J. (2002). Globalización e interdisciplinariedad: el currículum integrado. Madrid: Morata.

Messina, G., Pieck E. \& Castañeda, E. (2008). Educación y trabajo: lecciones desde la práctica innovadora en América Latina. Santiago de Chile: OREALC/UNESCO.

Ministerio de Educación y Cultura (1993). Diseño Nacional de Educación. Reforma Educativa. Asunción: MEC.

Ministerio de Educación y Cultura. (2011). La Educación Técnica y Profesional en el Paraguay. Antecedentes, contexto y perspectivas. Asunción: MEC.

Ministerio de Educación y Cultura. (2015). Seguimiento a egresados egresadas de la Educación Media. Bachillerato Técnico en Contabilidad. Bachillerato Técnico Agropecuario. Bachillerato Técnico en Electricidad. Asunción: Centro de Investigación e Innovación Educativa (CIIE). 


\section{Rev. cient. estud. investing. 6(1), 78-87; setiembre 2017}

Ministerio de Justicia y Trabajo, Ministerio de Industria y Comercio Ministerio de Agricultura y Ganadería, Ministerio de Obras Públicas y Comunicaciones y Ministerio de Salud Pública y Bienestar Social. (2011). Plan Nacional de Mejoramiento de la Educación Técnica y Profesional en el Paraguay 2011-2013. Asunción: MEC.

Stenhouse, L. (1987). La investigación como base de la enseñanza. Madrid: Morata.

\section{SOBRE LOS AUTORES}

Alcira Sosa es Licenciada Ciencias de la Educación y M.Sc. en Gestión Educativa. Docente universitaria. Se ha desempeñado como directora y catedrática en colegios secundarios. Ha ocupado cargos en el Ministerio de Educación de Paraguay en los ámbitos de Educación Media, Cooperación Internacional y Formación Continua de los Educadores.

Sara López es Licenciada Trabajo Social; M. Sc. Gestión Educativa; M. Sc. Evaluación Calidad de la Educación, M.Sc. Trabajo Social; Doctoranda en Ciencias Humanas y Sociales. Docente de grado y posgrado en universidades públicas y privadas. Ha ocupado cargos en gestión universitaria y en gestión del MEC. Forma parte de equipos de investigación. Ha realizado publicaciones a nivel nacional e internacional.

María Victoria Zavala es Licenciada Ciencias de la Educación, M.Sc. Educación, énfasis Investigación. Doctoranda en Educación. Se ha desempeñado como directora en Institución de Educación Superior. Se desempeña como docente: Escuela de Posgrado (UNE) y Universidad Iberoamericana (UNIBE). Miembro del equipo de Investigador UNIBE y de UNE. Tutoría de tesis.

Hugo Speratti es Licenciada Psicología, M Sc. Investigación, Ciencias Sociales; Doctorando Educación, Esp. Licitaciones Internacionales, proyectos de cooperación. Se desempeña como Docente: Escuela de Posgrado (UNE) y Universidad Iberoamericana (UNIBE), IFD Nuestra Señora de la Asunción. Miembro del equipo de Investigador (UNIBE) y Coordinador Oficina RRII. En Psicología UNA, Coordinador Autoevaluación.

Graciela Molinas es Lic. Ciencias de la Educación, M.Sc. Gestión Educativa. Doctoranda en Educación. Se desempeña como Coordinadora proyectos de investigación (UNIBE-CONACYT). Docente investigadora UNE. Docente: Escuela de Posgrado (UNE) y (UNIBE), Instituto de Trabajo Social (ITS), Carrera de Música (FADA). Posgrado, Facultad de Filosofía y Ciencias Humanas (FF y CH), UCA Tutora de tesis.

Claudio Suasnabar es Doctor Ciencias Sociales, Posdoctorado Educación, M.Sc. Magister Ciencias Sociales Orientación en Educación. Docente Universidad Nacional de La Plata, Universidad Nacional de Rosario. Docente Investigador Categoría I el en Programa de Incentivos a la Investigación, Secretaría de Políticas Universitarias del Ministerio de Cultura y Educación de la Nación (SPU). 
Educación técnica agropecuaria e industrial y el sector productivo. Sosa et al.

Coordinador de cursos internacionales en Maestrías y doctorados.

Rodolfo Elías es Licenciado Psicología, M. Sc. en Psicología Social y en Políticas Educativas. Se ha desempeñado como Investigador (CPES), Coordinación coordinó proyectos sociales para UNICEF-Paraguay Organización de Estados Iberoamericanos (OEI). Actualmente es investigador del área de Educación del Centro "Investigación para el Desarrollo" (ID) y Universidad Iberoamericana (UNIBE) y coordinador académico FLACSO Paraguay.

\section{Como citar}

Sosa, A., López, S. R., Zavala, M. V., Speratti, H., Molinas, G. (2017). Un acercamiento al diálogo entre la educación técnica agropecuaria e industrial y el sector productivo. Rev. cient. estud. investig., 6(1), 78-87. doi: 10.26885/rcei.6.1.78 